\title{
XII. Krystallographische Untersuchung der Dinitrobenzole und Nitrophenole.
}

Von

Th. V. Barker in Oxford.

(Mit 8 Textfiguren.)

1. Orthodinitrobenzol $\mathrm{C}_{6} \mathrm{H}_{4}\left(\mathrm{NO}_{2}\right)_{2}^{1,2}$.

Fig. 1.

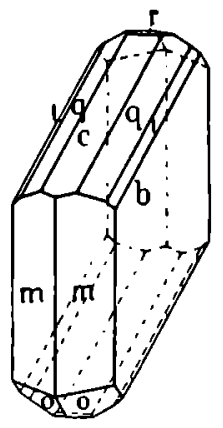

Diesen Körper hat bereits Bodewig (Pogg. Ann. 1876, 158, 239) goniometrisch untersucht.

Habitus: Tafelig nach der Symmetrieebene (Fig. 1).

Krystallsystem: Monoklin.

Axenverhältnis :

$a: b: c=0,6112: 1: 0,5735 ; \quad \beta=11207^{\prime}$.

Beobachtete Formen: $b\{010\}, m\{110\}, c\{001\}, q\{011\}$, $r\{T 02\}, o\{111\}$.

Ich erhielt Krystalle von Herrn Prof. Körner, an denen eine neue Form $t\{021\}$ beobachtet wurde.

Winkeltabelle (Bodewig).

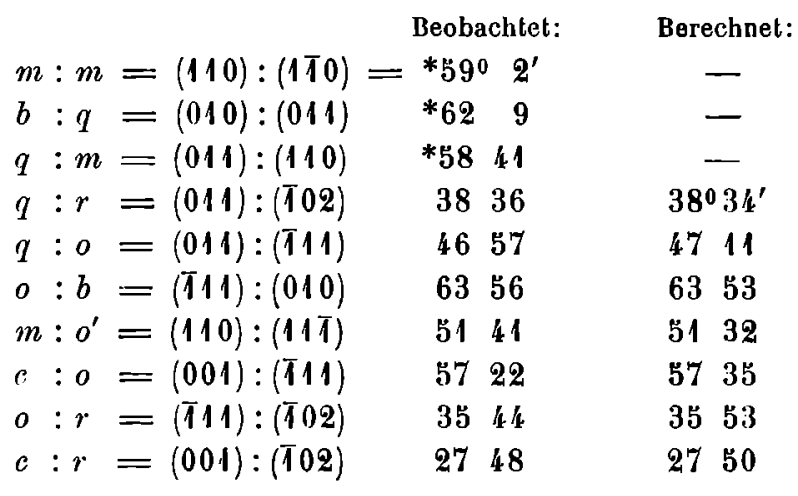


Krystallographische Untersuchung der Dinitrobenzole und Nitrophenole. 155

$$
\begin{array}{lll} 
& \text { Beobachtet: } & \text { Berechnet: } \\
c: m=(001):(110) & =70^{\circ} 56^{\prime} & 70^{0} 53^{\prime} \\
c: q=(001):(011) & 2751 & 2751 \\
b: t=(010):(021) & 4320 & 4325
\end{array}
$$

Spaltbarkeit unvollkommen nach der Basis.

Die optischen Eigenschaften wurden von E. Wichel (Dissertation Göttingen 1884; Ausz. diese Zeitschr. 11, 82) eingehend untersucht: Axenebene (010) für blau, senkrecht dazu für rot, negativ. $2 H_{a}=6^{\circ} 37^{\prime}(\mathrm{Li})$, $3^{0} 26^{\prime}(\mathrm{Na}), 5^{0} 23^{\prime}(\mathrm{Tl}), 14^{0}(\mathrm{Cu}) . \quad 2 V_{a}=8^{0} 30^{\prime}(\mathrm{Li}), 5^{0} 12^{\prime}(\mathrm{Na}), 7^{0} 11^{\prime}$ $(T l), 16^{\circ}(C u)$.

Eine Hauptschwingungsrichtung in der Symmetrieebene bildet für Gelb $65^{\circ} 48^{\prime}$ mit der Verticalaxe im spitzen Winkel $\beta$ (Bodewig).

Specifisches Gewicht bestimmt mit der Westphalschen Wage und Thouletschen Lösung bei $170=1,565$.

Molekularvolum: 107,42 .

Topische Axen: $\chi=4,2270, \psi=6,9159, \omega=3,9663$.

\section{Metadinitrobenzol $\mathrm{C}_{8} \mathrm{H}_{4}\left(\mathrm{NO}_{2}\right)_{2}^{1,3}$.}

Von Bodewig gemessen (loc. cit.).

Habitus: Tafelig nach $b\{010\}$ (Fig. 2).

Krystallsystem: Rhombisch.

Axenverhältnis :

$a: b: c=0,5302: 1: 0,2855$.

\begin{tabular}{|c|c|c|c|c|}
\hline$m: m$ & $=$ & $(110):(1 \bar{T} 0)$ & $\begin{array}{l}\text { Beobach tet: } \\
=55^{0} 47^{\prime}\end{array}$ & $\begin{array}{c}\text { Berechnet } \\
55^{0} 52^{\prime}\end{array}$ \\
\hline$n: n$ & $=$ & $(120):(1 \overline{2} 0)$ & 9320 & 9322 \\
\hline$k: k$ & $=$ & $(140):(1 \pi 0)$ & 12932 & 12930 \\
\hline$l: l$ & $=(1$ & $(14.0):(1 . \overline{4} .0)$ & $164 \quad 28$ & $164 \quad 40$ \\
\hline$: q$ & $=$ & $(010):(011)$ & $* 74 \quad 4$ & - \\
\hline$: r$ & $=$ & $(100):(101)$ & $*_{61}^{*} \quad 42$ & - \\
\hline$: r$ & $=$ & $(011):(101)$ & $32 \quad 12$ & 320 \\
\hline$r: k$ & $=$ & $(101):(140)$ & 7816 & 7820 \\
\hline$r: n$ & $=$ & $(101):(120)$ & 715 & 711 \\
\hline$r: m$ & $=$ & $(101):(110)$ & $6 !$ & 6514 \\
\hline
\end{tabular}

Beobachtete Formen: $b\{010\}, q\{011\}$, $r\{101\}, m\{110\}, n\{120\}, k\{140\}, l\{1.14 .0\}$.

Winkeltabelle (Bodewig).

Fig. 2.

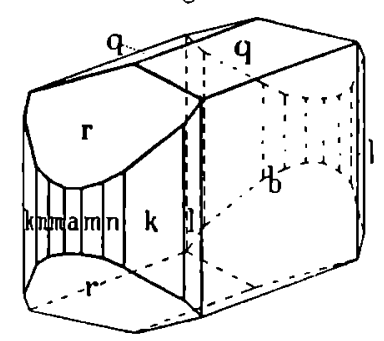

$55^{0} 52^{\prime}$

12930

$164 \quad 40$

Axenebene (100); Doppelbrechung negativ.

Spaltbarkeit: keine bemerkbar.

Spec. Gewicht bei $17^{\circ}=1,546$. Molekularvolumen: 108,70.

Topische Axen: $\chi=8,9549, \psi=4,7479, \omega=2,5566$. 


\section{Paradinitrobenzol $\mathrm{C}_{6} \mathrm{H}_{4}\left(\mathrm{NO}_{2}\right)_{2}^{1,4}$.}

Von Bodewig gemessen.

Habitus: Prismatisch infolge einer Verlängerung nach der verticalen Axe.

Fig. 3 .

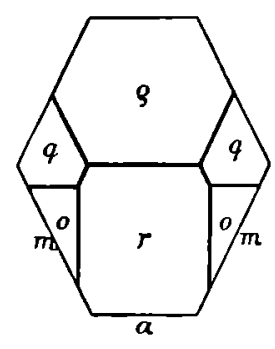

Krystallsystem: Monoklin.

Axenverhältnis :

$$
a: b: c=2,0383: 1: 1,0432 \text {; }
$$$$
\beta=92018^{\prime} \text {. }
$$

Beobachtete Formen:

$$
\begin{gathered}
a\{100\}, m\{110\}, r\{101\}, \\
\varrho\{101\}, q\{011\} .
\end{gathered}
$$

Ich erhielt ein Präparat von Herrn Prof. Körner und fand eine neue Form $o\{111\}$ an Krystallen, die aus Alkohol

erhalten wurden; der Habitus dieser Krystalle ist in Fig. 3 gegeben.

Winkeltabelle (Bodewig).

$\begin{array}{lcc}m: m=(110):(1 \overline{1} 0) & \begin{array}{c}\text { Beobachtet: } \\ 127037^{\prime}\end{array} & \begin{array}{c}\text { Berechnet: } \\ 127042^{\prime}\end{array} \\ a: m=(100):(110) & * 6351 & - \\ a: r=(100):(101) & { }^{*} 615 & - \\ a: \varrho=(100):(10 T) & 6442 & 6443 \\ r: \varrho^{\prime}=(101):(T 01) & * 5412 & - \\ m: r=(110):(101) & 7743 & 7742 \\ r: q=(101):(011) & 5138 & 5146 \\ q: q=(011):(0 T 1) & - & 9222 \\ m: q=(110):(011) & 4845 & 4842 \\ m: q=(\overline{1} 10):(011) & 5046 & 5032 \\ r: o=(101):(111) & 4220 & 4224 \\ o: \varrho^{\prime}=(111):(101) & 6417 & 6424\end{array}$

Axenebene (010); eine Hauptschwingungsrichtung für Gelb macht mit der Verticalaxe einen Winkel von $38^{\circ} 30^{\prime}$ im stumpfen Axenwinkel; durch $a$ und $r$ ist je eine Axe sichtbar (Bodewig).

Spaltbarkeit unvollkommen nach $a$, sehr vollkommen nach $r$ und $r^{\prime}$.

Spec. Gewicht bei $17^{0}=1,587$. Molekularvolumen: 105,89.

Topische Axen: $\chi=7,5011, \psi=3,6801, \omega=3,8391$. 


\section{Orthonitrophenol $\mathrm{C}_{6} \mathrm{H}_{4}(\mathrm{OH}) \stackrel{2}{\mathrm{~N}} \mathrm{O}_{2}$.}

Von Groth gemessen (Ber. d. d. chem. Ges. 1870, 3, 451).

Habitus: Prismatisch.

Krystallsystem: Rhombisch.

Axenverhältnis: $a: b: c=0,873: 1:(0,60$ ?).

Mehrere Versuche, Krystalle mit Endfächen zu erhalten, waren ganz erfolglos.

Spec. Gewicht bei $14^{0}=1,484$. Molekularvolumen: 93,70 .

5. Kaliumsalz des Orthonitrophenols $\mathrm{C}_{8} \mathrm{H}_{4}\left(\mathrm{O} \underset{\mathrm{K}}{\mathrm{N}} \stackrel{2}{\mathrm{~N}} \mathrm{O}_{2}\right.$. aq

(gemeinsam mit Herrn R. C. Spiller.)

Wegen der großen Tendenz, übersättigte wässerige Lösungen zu bilden, sind meßbare Krystalle dieses Salzes schwer zu erhalten. Trotzdem ist das Wachstum außerordentlich schön, wenn es im polarisierten Lichte beobachtet wird, und empfiehlt sich sehr zum Vorlesungszwecke.

Der Habitus ist in Fig. 4 gegeben; die mikroskopischen Krystalle sind prismatisch nach der Symmetrieaxe.

Krystallsystem: Monoklin.

Axenverhältnis:

$$
\begin{gathered}
a: b: c=2,3456: 1: 2,2691 ; \\
\beta=102^{\circ} 48^{\prime} .
\end{gathered}
$$

Fig. 4.

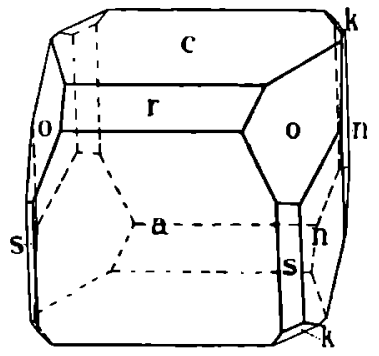

Beobachtete Formen: $a\{100\}, c\{001\}, r\{102\}, o\{111\}, n\{120\}$, $s\{320\}, \operatorname{lc}\{324\}$.

\begin{tabular}{|c|c|c|c|c|c|c|c|}
\hline & & & & & & & Dereconel: \\
\hline$a: c=(100):(001)$ & 21 & & $4^{\prime}-77$ & $017^{\prime}$ & & & - \\
\hline$: r=(100):(102)$ & 19 & 53 & $58-54$ & 18 & 54 & 7 & 540 \\
\hline$: c=(102):(001)$ & 19 & 22 & $48-23$ & 15 & *23 & $4 \frac{1}{2}$ & - \\
\hline$: s=(100):(320)$ & 5 & 57 & $4-57$ & 17 & 57 & $10^{\circ}$ & $56 \quad 45$ \\
\hline$: n=(100):(120)$ & 9 & 77 & $25-78$ & 16 & 77 & 48 & 7740 \\
\hline$: 0=(100):(111)$ & 25 & 62 & $56-64$ & 24 & $*_{63}$ & 49 & - \\
\hline$: k=(\bar{T} 00):(\overline{3} 24)$ & 5 & 70 & $32-71$ & 15 & 70 & 55 & 71 \\
\hline$: o=(102):(111)$ & 17 & 56 & $6-57$ & 2 & 56 & 45 & 5630 \\
\hline$n=(102)$ & 7 & 82 & $40-83$ & 22 & 82 & 49 & 8249 \\
\hline$k=(102):$ & 1 & & - & & 71 & 49 & 7129 \\
\hline$: o=(001):(111)$ & 12 & 62 & $4.4-63$ & 41 & 63 & 24 & 6313 \\
\hline$n=(001):(120)$ & 6 & 87 & $3-88$ & 0 & 87 & 27 & 8718 \\
\hline
\end{tabular}

Winkeltabelle. 


\begin{tabular}{lccccc} 
& Nr. & Grenzen: & \multicolumn{2}{c}{ Mittel: } & Berechnet: \\
$c: s=(001):(320)$ & 1 & - & $8209^{\prime}$ & $83^{0} 1^{\prime}$ \\
$c: k=(001):(\overline{3} 24)$ & 1 & - & 5750 & 5725 \\
$o: o=(111):(\bar{T} 1 T)$ & 5 & $68^{0} 43^{\prime}-69^{0} 40^{\prime}$ & 6913 & 6936 \\
$o: n=(111):(\bar{T} 20)$ & 3 & $4420-4519$ & 4444 & 4455 \\
$n: k=(120):(\overline{3} 24)$ & 1 & - & 514 & 5122 \\
$n: k=(\overline{1} 20):(\overline{3} 24)$ & 1 & - & 3937 & 4018
\end{tabular}

Spaltbarkeit vollkommen nach $a(100)$.

Axenebene (010); durch $a$ und $e$ ist je eine Axe sichtbar; die Mittellinie (wahrscheinlich erste), die schief durch $r$ heraustritt, ist negativ. Axenwinkel für blau viel größer als für rot; Doppelbrechung außerordentlich stark.

Spec. Gewicht bei $20^{\circ}=1,657$. Molekularvolumen: 117,80.

Topische $\Lambda$ xen: $\chi=6,6412, \psi=2,8314, \omega=6,4246$.

\section{Metanitrophenol $\mathrm{C}_{6} \mathrm{H}_{4}(\mathrm{OH}) \stackrel{3}{\mathrm{~N}} \mathrm{O}_{2}$.}

Dieser Körper soll früher von Fels (diese Zeitschr. 1900, 32, 374) gemessen und zwar hexagonal gefunden worden sein. Bei seinem gemessenen Präparate fand ich aber nicht den richtigen Schmelzpunkt; es war also nicht Metanitrophenol.

lig. : 5.

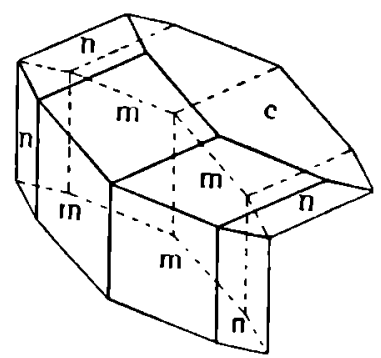

Das Präparat, das zur krystallographischen Untersuchung diente, war von mir selbst nach dem gewöhnlichen Verfahren aus Metanitranilin dargestellt.

Habitus: Tafelig nach der Basis.

Krystallsystem: Monoklin.

Axenverhältnis:

$$
a: b: c=1,8446: 1: \text { ?; } \beta=120^{\circ} 21^{\prime} \text {. }
$$
$m\{110\}$.

Beobachtete Formen: $c\{001\}, n\{210\}$,

Die Krystalle waren fast immer Zwillinge nach $c(001)$ (Fig. 5).

Winkeltabelle.

Beobachtet:

$c: m=(001):(110)=74020^{\prime}$

Berechnet:

$c: n=(001):(210)$

*66 43

$n: n=(210):(2 T 0) \quad * 77 \quad 2$

$m: m=(110):(1 \overline{1} 0)$

1934

11545

$n: m=(210):(110)$

1921

$n: n^{\prime}=$ (Zwilling)

$47 \quad 1$

$46 \quad 34$ 
Eine Hauptschwingungsrichtung macht mit der Verticalaxe einen Winkel von $58^{\circ} 30^{\prime}$ im stumpfen Axenwinkel $\beta$. Doppelbrechung schwach.

Spaltbarkeit vollkommen nach der Basis, weniger vollkommen nach der Symmetrieebene.

Spec. Gewicht bei $20^{\circ}=1,485$. Molekularvolumen: 93,64 .

\section{Paranitrophenol $\mathrm{C}_{6} \mathrm{H}_{4}(\mathrm{O} \stackrel{\mathrm{H}}{\mathrm{H}}) \stackrel{4}{\mathrm{~N}} \mathrm{O}_{2}$.}

Krystalle dieses Kürpers hat zuerst Kokscharow untersucht (Bull. phys. math. St. Pétersbourg 1858, 17), der zwei Formen beschrieb; später hat Lehmann (diese Zeitschr. 1877, 1, 45) die Dimorphie dieses Kürpers bestätigt.

Fig. 6.

\section{Labile Modiflcation.}

Die untersuchten Krystalle waren aus Toluol bei Temperaturen höher als $65^{\circ}$ erhalten.

Habitus: Kurzprismatisch (Fig. 6).

Krystallsystem: Monoklin.

Axenverhältnis:

$$
a: b: c=0,3398: 1: 1,3836 ; \beta=106^{\circ} 5^{\prime} \text {. }
$$
$a\{100\}$.

Beobachtete Formen: $q\{011\}, b\{010\}, m\{110\}$,

\section{Winkeltabelle.}

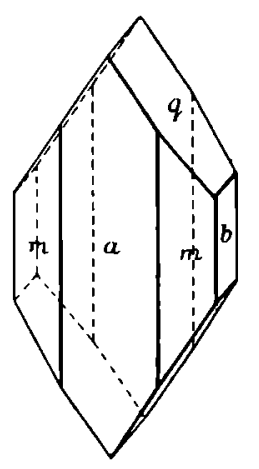

Beobachtet: Berechnet:

$q: q=(011):(0 \overline{1} 1)=* 105^{0} 51^{\prime} 40^{\prime \prime}$

$b: m=(010):(110) \quad * 715930$

$m: q=(110):(011) \quad * 653430$

$m: q=(110):(0 \bar{T} 1)$

9430

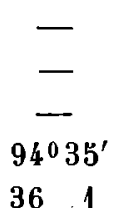

Koksch. Beob.: $105^{\circ} 23^{\prime}$

$m: m=(110):(1 \bar{T} 0)$

$36 \quad 2$

361

$65 \quad 23$

$94 \quad 34$

$36 \quad 15$

Spallbarkeit nach der Symmetrieebene unvollkommen.

Spec. Gewicht bei $16^{\circ}=1,495$. Molekularvolumen: 93,01.

Topische Axen: $\chi=2,0093, \psi=5,9132, \omega=8,1821$.

\section{Stabile Modification.}

Krystalle aus Toluol bei Temperaturen niedriger als $65^{\circ}$.

Habitus: Kurzprismatisch mit $o\{121\}$ gut ausgebildet.

Krystallsystem: Monoklin.

Axenverhältnis :

$$
a: b: c=0,6594: 1: 0,8416 ; \beta=53^{0} 24^{\prime} 30^{\prime \prime} .
$$

Beobachtete Formen: $o\{121\}, m\{110\}, a\{100\}, b\{010\}, c\{001\}, r\{101\}$. 
Einen typischen Krystall aus Toluol stellt Fig. 7 dar; Fig. 8 einen aus wässeriger Lösung erhaltenen Krystall (Kokscharow).

Fig. 7.

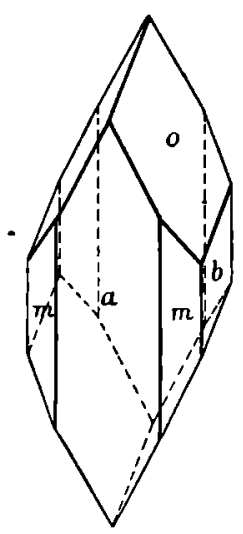

Fig. 8.

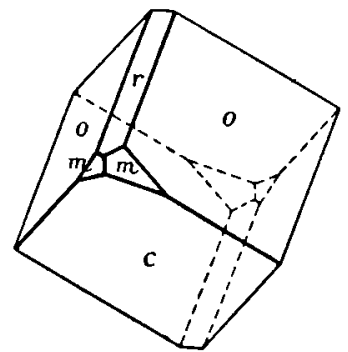

Winkeltabelle.

Barker.

Kokscharow.

Beobachtet: Berechnet: Beobachtet: Berechnet:

\begin{tabular}{|c|c|c|c|c|}
\hline$m: m=(110):(1 \pi 0)$ & $=* 55^{0} 48^{\prime}$ & - & $* 55^{0} 42^{\prime}$ & 一 \\
\hline$o: o=(121):(1 \overline{2} 1)$ & $* 104 \quad 11$ & 一 & $* 104 \quad 24$ & 一 \\
\hline$: o=(100):(121)$ & *66 36 & - & - & - \\
\hline$m: o=(110):(T 2 T)$ & 8858 & $89^{\circ} 0^{\prime}$ & 8851 & 8836 \\
\hline$m: o=(110):(121)$ & 441 & 4357 & 441 & 442 \\
\hline$c: o=(001):(121)$ & - & 8158 & $* 8151$ & - \\
\hline$c: r=(001):(101)$ & - & 7651 & 7655 & 7638 \\
\hline$c: m=(001):(110)$ & - & $121 \quad 47$ & 12151 & 1221 \\
\hline
\end{tabular}

Meine berechneten Werte stimmen also ganz gut mit den von Kokscharow beobachteten überein.

Axenebene (010); die erste Mittellinie macht mit der Verticalaxe einen Winkel von $2^{0}$ im stumpfen Axenwinkel (Lehmann); Doppelbrechung sehr stark.

Spaltbarkeit nach dem Prisma vollkommen.

Spec. Gewicht bei $20^{\circ}=1,479$.

Die topischen Axen wurden nach den folgenden Formeln berechnet:

$$
\begin{aligned}
& \psi=\sqrt[3]{\frac{2 V}{a c \sin \beta}}, \quad x=a \psi, \quad \omega=c \psi \\
& x=4,9460, \quad \psi=7,5008, \quad \omega=6,3126 .
\end{aligned}
$$


Krystallographische Untersuchung der Dinitrobenzole und Nitrophenole. 161

\section{Dinitrophenole $\mathrm{C}_{6} \mathrm{H}_{3}(\mathrm{OH})\left(\mathrm{NO}_{2}\right)_{2}$.}

Das $a$-Dinitrophenol ist von Fels gemessen. Ich habe seine Messungen bestätigt und eine neue Form $r\{101\}$ beobachtet.

$$
\begin{array}{lcc} 
& \text { Beobachtet: } & \text { Berechnet: } \\
r: m=(101):(110)= & 45^{0} 48^{\prime} & 45^{\circ} 58^{\prime} \\
r: t=(101):(032) & 5919 & 5928
\end{array}
$$

Spaltbarkeit sehr unvollkommen nach $m$ (Fels) und $t$ (Barker).

Trotz vieler Bemühungen konnte ich nicht meßbare Krystalle der anderen Dinitrophenole beschaffen.

Zum Schlusse ist es mir eine angenehme Pflicht, meinem hochverehrten Lehrer, Herrn Prof. Groth, unter dessen Leitung vorliegende Untersuchung im Winter 1902-1903 entstand, auch an dieser Stelle meinen aufrichtigsten Dank auszusprechen.

Mineralogisches Institut

- München.
University Museum

Oxford. 